\section{Exercise programs for people with dementia}

\author{
Dorothy Forbes, Emily J. Thiessen, Catherine M. Blake, \\ Scott S. Forbes, Sean Forbes
}

The independent commentary was written by

Maysa Seabra Cendoroglo

\begin{abstract}
BACKGROUND: This is an update of our previous 2008 review. Several recent trials and systematic reviews of the impact of exercise on people with dementia are reporting promising findings.

OBJECTIVE:

Primary: Do exercise programs for older people with dementia improve cognition, activities of daily living (ADLs), challenging behaviour, depression, and mortality in older people with dementia? Secondary: Do exercise programs for older people with dementia have an indirect impact on family caregivers' burden, quality of life, and mortality? Do exercise programs for older people with dementia reduce the use of healthcare services (e.g. visits to the emergency department) by participants and their family caregivers?
\end{abstract}

METHODS:

Search methods: We identified trials for inclusion in the review by searching ALOIS (www.medicine.ox.ac.uk/alois), the Cochrane Dementia and Cognitive Improvement Group's Specialised Register, on 4 September 2011, and again on 13 August 2012. The search terms used were: 'physical activity' OR exercise OR cycling OR swim* OR gym* OR walk* OR danc* OR yoga OR 'tai chi'.

Selection criteria: In this review, we included randomized controlled trials in which older people, diagnosed with dementia, were allocated either to exercise programs or to control groups (usual care or social contact/activities) with the aim of improving cognition, ADLs, behaviour, depression, and mortality. Secondary outcomes related to the family caregiver(s) and included caregiver burden, quality of life, mortality, and use of healthcare services.

Data collection and analysis: Independently, at least two authors assessed the retrieved articles for inclusion, assessed methodological quality, and extracted data. Data were analysed for summary effects using RevMan 5.1 software. We calculated mean differences or standardized mean difference (SMD) for continuous data, and synthesized data for each outcome using a fixed-effect model, unless there was substantial heterogeneity between studies, when we used a random-effects model. We planned to explore heterogeneity in relation to severity and type of dementia, and type, frequency, and duration of exercise program. We also evaluated adverse events.

MAIN RESULTS: Sixteen trials with 937 participants met the inclusion criteria. However, the required data from three trials and some of the data from a fourth trial were not published and not made available. The included trials were highly heterogeneous in terms of subtype and severity of participants' dementia, and type, duration and frequency of exercise. Only two trials included participants living at home. Our metaanalysis suggested that exercise programs might have a significant impact on improving cognitive functioning (eight trials, 329 participants; SMD $0.55,95 \%$ confidence interval (CI) 0.02 to 1.09). However, there was substantial heterogeneity between trials (I 2 value $80 \%$ ), most of which we were unable to explain. We repeated the analysis omitting one trial, an outlier, that included only participants with moderate or severe dementia. This reduced the heterogeneity somewhat (12 value 68\%), and produced a result that was no longer significant (seven trials, 308 participants; SMD 0.31, 95\% Cl -0.11 to 0.74). We found a significant effect of exercise programs on the ability of people with dementia to perform ADLs (six studies, 289 participants; SMD 0.68, 95\% Cl 0.08 to 1.27). However, again we observed considerable unexplained statistical heterogeneity ( 12 value $77 \%$ ) in this meta-analysis. This means that there is a need for caution in interpreting these findings. In further analyses, we found that the burden experienced by informal caregivers providing care in the home may be reduced when they supervise the participation of the family member with dementia in an exercise program (one study, 40 participants; MD $-15.30,95 \% \mathrm{Cl}-24.73$ to -5.87 ), but we found no significant effect of exercise on challenging behaviours (one study, 110 participants; MD $-0.60,95 \% \mathrm{Cl}-4.22$ to 3.02 ), or depression (six studies, 341 participants; MD $-0.14,95 \% \mathrm{Cl}-0.36$ to 0.07 ). We could not examine the remaining outcomes, quality of life, mortality, and healthcare costs, as either the appropriate data were not reported, or we did not retrieve trials that examined these outcomes.

AUTHORS' CONCLUSIONS: There is promising evidence that exercise programs can have a significant impact in improving ability to perform ADLs and possibly in improving cognition in people with dementia, although some caution is advised in interpreting these findings. The programs revealed no significant effect on challenging behaviours or depression. There was little or no evidence regarding the remaining outcomes of interest.

This is the abstract of a Cochrane Review published in the Cochrane Database of Systematic Reviews (CDSR) 2013, issue 12, Art. No.: CD006489. DOI: 10.1002/14651858.CD006489.pub3 (http://onlinelibrary.wiley.com/doi/10.1002/14651858.CD006489.pub3/abstract;jsessi onid=EB059310003BD3AC29CC6D77710D8779.f01 t02). For full citation and authors' details see reference 1 .

The full text is available from: http://onlinelibrary.wiley.com/ doi/10.1002/14651858.CD006489.pub3/abstract.

The abstract is also available in the Portuguese, French and Spanish languages from: http://summaries.cochrane.org/CD006489/exerciseprograms-for-people-with-dementia.

\section{REFERENCE}

1. Forbes D, Thiessen EJ, Blake CM, Forbes SC, Forbes S. Exercise programs for people with dementia. Cochrane Database Syst Rev. 2013;12:CD006489.

\section{COMMENTS}

This systematic review published by Forbes et al. is a continuation and update of the study that they published in 2008. It aimed to identify the benefits of physical exercise for elderly people with impaired cognition. The review included randomized studies with control groups. The quality of the studies was very well defined and there was an interest in selecting studies in which the intervention consisted of physical activity unassociated with other forms of treatment.

In the systematic review conducted in 2008, a meta-analysis was conducted and the results were insufficient to show any evidence of benefits from exercise in relation to the primary objectives. In the study of 2013, it was observed that the subjects presented improved ability to carry out activities of daily living, possibly with improved cognition. In relation to the other primary and secondary objectives, the data were insufficient or inconclusive. 
In preclinical studies, the action of exercise has been seen to involve structural and functional changes to neurons. Exercise-related neurogenesis and neuron remodeling have been observed through increased production of brain-derived synaptic proteins (BDNF). However, neuroplasticity also results from the action of other factors involving serotonin reuptake, $\mathrm{N}$-methyl-D-aspartate (NMDA) receptors and calorie and cholesterol restriction; and from manipulation of cerebral magnetic fields and electrical activity. Considering that it is possible and likely that interactions between multiple factors result in modifications to the response to the treatment used, it can be comprehended that such interactions make it difficult to identify the effect of one intervention alone. In this regard, these authors' rigorous methodological care has placed value on their findings and partly explains the lack of evidence relating to the other objectives.

Maysa Seabra Cendoroglo. Adjunct Professor in the Discipline of Geriatrics and Gerontology, Universidade Federal de São Paulo (Unifesp), São Paulo, Brazil. 